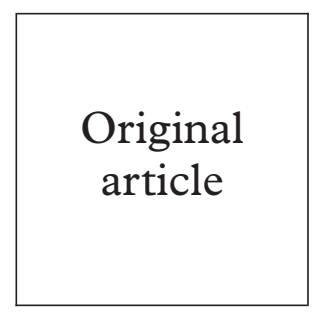

London School of Hygiene and Tropical Medicine, London, UK D Watson-Jones

P Mayaud

J Todd

B West

H Grosskurth

R Hayes

D Mabey

Municipal Office of Health, Mwanza,

Tanzania

K Mugeye

L Ndeki

African Medical and Research Foundation (AMREF), Mwanza, Tanzania

P Mayaud

Bernadette

Cleophas-Frisch

Institute of Tropical Medicine, Antwerp,

Belgium

A Buvé

M Laga

National Institute of Medical Research,

Mwanza, Tanzania

D Watson-Jones

J Todd

F Mosha

B West

Correspondence to:

Professor Mabey

d.mabey@1shtm.ac.uk

Accepted for publication 7 June 2000

\title{
High prevalence of trichomoniasis in rural men in Mwanza, Tanzania: results from a population based study
}

\author{
Deborah Watson-Jones, Kokungoza Mugeye, Philippe Mayaud, Leonard Ndeki, \\ James Todd, Frank Mosha, Beryl West, Bernadette Cleophas-Frisch, Heiner Grosskurth, \\ Marie Laga, Richard Hayes, David Mabey, Anne Buvé
}

Objectives: To measure the prevalence of urethral infections including trichomoniasis in rural
Tanzanian men, to assess the prevalence of symptoms and signs among men with Trichomonas vaginalis, and to analyse the risk factors for trichomoniasis.

Design: A cross sectional study of 1004 men aged 15-54 years in a rural community in north west Tanzania.

Methods: Participants were interviewed about sexual behaviour and symptoms of sexually transmitted diseases. First fraction urine samples and urethral swabs were collected and used to test for $T$ vaginalis by wet preparation and culture, Neisseria gonorrhoeae by culture, Chlamydia trachomatis by ligase chain reaction and non-specific urethritis by Gram stain. Urine was also tested for the presence of leucocytes using a leucocyte esterase dipstick. Men were re-interviewed 2 weeks later to document new symptoms and signs of urethritis.

Results: Complete laboratory results were available on 980 men. One in four men had laboratory evidence of urethritis. T vaginalis was found in 109 individuals (11\%), gonorrhoea in eight $(0.8 \%)$, and chlamydial infection in $15(1.5 \%)$. Over $50 \%$ of men with urethritis were asymptomatic. The prevalence of signs and symptoms was similar among men with $T$ vaginalis alone compared with men with other urethral infections. The sensitivity and specificity of the leucocyte esterase dipstick (LED) test for detecting $T$ vaginalis were $80 \%$ and $48 \%$ respectively in symptomatic men and $60 \%$ and $68 \%$ in asymptomatic men. Factors associated with trichomoniasis included religion, type of employment, and marital status.

Conclusions: A high prevalence of urethritis was found in men in this community based study. More than half of the urethral infections detected were asymptomatic. The most prevalent pathogen was $T$ vaginalis. Studies are needed on the prevalence of trichomoniasis in men presenting to health services with complaints suggestive of urethritis since treatment for $T$ vaginalis is not included in the syndromic management of urethritis in most countries. The performance of the LED test as a screening test for trichomoniasis was unsatisfactory in both symptomatic and asymptomatic men. Improved screening tests are urgently needed to identify urethral infections that are asymptomatic and which are not covered by current syndromic management algorithms. (Sex Transm Inf 2000;76:355-362)

Keywords: urethritis; Tanzania; Trichomonas vaginalis

\section{Introduction}

Both ulcerative and non-ulcerative sexually transmitted diseases (STDs) are cofactors for the transmission of the human immunodeficiency virus (HIV).$^{1-4}$ Improved case detection and management of patients presenting to health services with symptoms and signs suggestive of STDs, has been shown to reduce the transmission of HIV in a randomised community based study. ${ }^{5}$ Any approach which relies on the self reporting of symptoms has the disadvantage that asymptomatic STDs will fail to be identified and treated and will therefore remain as a potentially infective pool in the population so that there may be little impact on the overall prevalence of STDs in the community.

Little is known about the prevalence of asymptomatic STDs in the community in subSaharan Africa and there have been few attempts to measure the proportion of both symptomatic and asymptomatic urethral infections in men from the general population. A previous study in Mwanza Region had found that at least $2.7 \%$ of men in the general population were infected with Neisseria gonorrhoeae and/or Chlamydia trachomatis and that the majority of these infections were asymptomatic. ${ }^{6}$ Since diagnostic tests were only performed on men who had symptoms of urethral discharge or genital ulceration or who had a positive urine screening test for leucocytes, the leucocyte esterase dipstick (LED) test, these results may have underestimated the prevalence of infection. Furthermore, studies of the aetiology of urethritis in sub-Saharan Africa have usually focused on chlamydial and gonococcal urethritis..$^{6-8}$ There is little information on the prevalence of Trichomonas vaginalis infection in men in this region although trichomoniasis is known to be one of the most prevalent STDs in women, ${ }^{9}{ }^{10}$ and in one study trichomoniasis was associated with an increased risk of HIV transmission. ${ }^{3}$ In male Kenyan transport workers trichomoniasis was more prevalent than either gonococcal or 
chlamydia urethritis $(6.0 \% v 3.4 \%$ and $3.6 \%$ respectively) and $83 \%$ of $T$ vaginalis infections were asymptomatic. ${ }^{11}$ There have been no studies that have examined the prevalence and risk factors associated with $T$ vaginalis infection in men from the general population in sub-Saharan Africa.

The present study was part of a set of studies conducted to assess the effectiveness of different strategies of case detection and management of STDs. The main objective was to measure the prevalence of urethral infections including trichomoniasis among rural Tanzanian men in the community, to assess the prevalence of symptoms and signs among men with urethral infection, and to assess the risk factors associated with trichomoniasis.

Identifying asymptomatic infections so that they might be adequately treated is problematic in developing countries since access to laboratory testing is generally limited. Strategies to overcome this have concentrated on risk assessment scores ${ }^{912}{ }^{13}$ and screening tests such as the LED test, which tests for leucocytes in first fraction urine and which has the advantages of being affordable (approximately $£ 0.23$ per test) and giving an immediate and easily interpretable result. The usefulness of the LED test in screening for male urethritis has been tested in selected populations such as male STD clinic attenders ${ }^{814}$ and in truck drivers in Kenya. ${ }^{11}$ We tested the sensitivity, specificity and positive predictive value of the LED test for the detection of $T$ vaginalis in men in the general population.

\section{Methods}

STUDY POPULATION AND STUDY DESIGN

A cross sectional community based study was carried out in Misungwi area, $40 \mathrm{~km}$ south of Mwanza town in north west Tanzania. Adult men living within a $3 \mathrm{~km}$ radius of the Misungwi health centre comprised the study population. The households in Tanzania are grouped into 10 household administrative units known as the "balozi", each of which has a balozi leader. Units of 10-15 balozis are further aggregated into a "kitongoji." The kitongoji leaders and study team visited each balozi head to draw up a list of every eligible man in that balozi. All men aged 15-54 years were then visited at home by the kitongoji and balozi leaders and a member of the study team and were asked to attend a field station located near their area of residence where a team of trained interviewers, laboratory technicians, and a clinician were based.

After obtaining informed consent, men who agreed to participate were interviewed on sociodemographic variables, sexual behaviour, symptoms of STD infections, and health seeking behaviour for STDs. A first fraction urine sample was collected at least 2 hours after they had last passed urine. The men were then interviewed by a clinician who asked them about symptoms of STDs. After a genital examination, including milking of the urethra if no discharge was seen, two urethral swabs were taken.
All men with symptoms of an STD plus a positive LED test or who had signs of an STD were treated at this visit using Tanzanian national guidelines. This included metronidazole only if $T$ vaginalis was seen on the wet preparation. They were also asked to notify their sexual partners of the preceding 3 months and were given contact slips for their partners to present for treatment at the health centre. Men found to have urinary schistosomiasis were treated with praziquantel. All subjects were asked to return to the study team at the health centre 2 weeks later where they had a further interview to document new symptoms and signs and then were given treatment for any STDs identified by the laboratory tests from their first visit. For men who had failed to attend for the first visit at least two home visits were made by a member of the study team and the balozi leader to encourage them to attend the field station when convenient.

LABORATORY METHODS

First fraction urine samples were tested with a urine dipstick test (LED Nephur test + Leuco, Boehringer-Mannheim, Lewes, Sussex) and read after 2 minutes for the presence of leucocytes and erythrocytes according to the manufacturer's instructions. A reading greater than "trace" was defined as a positive LED. A $10 \mathrm{ml}$ aliquot from each urine sample was centrifuged at $3000 \mathrm{rpm}$ for 10 minutes. A drop of the resuspended pellet was put onto a glass slide as a wet mount preparation and examined under light microscopy for the detection of motile protozoa with the characteristic morphology of $T$ vaginalis and also for the eggs of Schistosoma haematobium. A swab of the pellet was used to inoculate a commercial culture medium for $N$ gonorrhoeae (Gonoline, bioMérieux, Marcyl'Etoile, France) which was incubated initially in the field at $36^{\circ} \mathrm{C}$, then transported to Mwanza and inspected after 48 and 72 hours. Positive cultures were identified by colony morphology, Gram stain, positive oxidase and catalase reactions, and the Phadebact agglutination test (Launch Diagnostics, Longfield, $\mathrm{UK})$.

A second swab from the pellet was used to inoculate a culture medium for $T$ vaginalis (InPouch TV, BioMed Diagnostics, San Jose, CA, USA). This was incubated at $36^{\circ} \mathrm{C}$ in the field, transported to the reference laboratory incubator in Mwanza, and examined under light microscopy for the presence of characteristic motile trichomonads at 3 and 7 days. Men were considered to be infected with $T$ vaginalis if either the wet preparation or the culture was positive for the organism. A $5 \mathrm{ml}$ aliquot of urine was stored in a cryotube at $-20^{\circ} \mathrm{C}$ before being transported to London for $C$ trachomatis testing by the ligase chain reaction (LCR, Abbott Laboratories, Abbott Park, IL, USA) following the manufacturer's instructions.

Urethral swabs were taken for $N$ gonorrhoeae culture using a second Gonoline and to make smears for Gram staining. Smears were examined under light microscopy for polymorphonuclear cells. Men were defined as having a specific urethral infection if any of the tests for 
Table 1 Prevalence of sexually transmitted diseases (STD) and schistosomiasis by age among 980 rural adult men, Tanzania

\begin{tabular}{|c|c|c|c|c|c|c|c|c|c|c|c|c|c|}
\hline \multirow{2}{*}{$\begin{array}{l}\text { Age } \\
\text { (years) }\end{array}$} & \multirow[b]{2}{*}{ No } & \multicolumn{2}{|c|}{$\begin{array}{l}\text { Trichomonas } \\
\text { vaginalis }\end{array}$} & \multicolumn{2}{|c|}{$\begin{array}{l}\text { Neisseria } \\
\text { gonorrhoeae * }\end{array}$} & \multicolumn{2}{|c|}{$\begin{array}{l}\text { Chlamydia } \\
\text { trachomatis* }\end{array}$} & \multicolumn{2}{|c|}{$\begin{array}{l}\text { Non-specific } \\
\text { urethritist }\end{array}$} & \multicolumn{2}{|c|}{ Urethritis $\neq$} & \multicolumn{2}{|c|}{$\begin{array}{l}\text { Schistosoma } \\
\text { haematobium }\end{array}$} \\
\hline & & No & $\%$ & No & $\%$ & No & $\%$ & No & $\%$ & No & $\%$ & $\mathrm{No}$ & $\%$ \\
\hline $15-19$ & 176 & 12 & $7 \%$ & 2 & $1 \%$ & 2 & $1 \%$ & 29 & $16 \%$ & 44 & $25 \%$ & 118 & $67 \%$ \\
\hline $20-24$ & 199 & 19 & $10 \%$ & 2 & $1 \%$ & 4 & $2 \%$ & 27 & $14 \%$ & 50 & $25 \%$ & 98 & $49 \%$ \\
\hline $25-29$ & 138 & 11 & $8 \%$ & 1 & $1 \%$ & 3 & $2 \%$ & 18 & $13 \%$ & 32 & $23 \%$ & 63 & $46 \%$ \\
\hline $30-34$ & 137 & 16 & $12 \%$ & 0 & $0 \%$ & 1 & $1 \%$ & 21 & $15 \%$ & 38 & $28 \%$ & 29 & $21 \%$ \\
\hline $35-44$ & 168 & 21 & $13 \%$ & 0 & $0 \%$ & 3 & $2 \%$ & 17 & $10 \%$ & 39 & $23 \%$ & 26 & $15 \%$ \\
\hline $45-54$ & 162 & 30 & $19 \%$ & 3 & $2 \%$ & 2 & $1 \%$ & 12 & $7 \%$ & 46 & $28 \%$ & 30 & $19 \%$ \\
\hline Total & 980 & 109 & $11 \%$ & 8 & $1 \%$ & 15 & $2 \%$ & 124 & $13 \%$ & 249 & $25 \%$ & 364 & $37 \%$ \\
\hline
\end{tabular}

*7 mixed infections: $3 T$ vaginalis $+N$ gonorrhoeae, $1 N$ gonorrhoeae $+C$ trachomatis, $3 T$ vaginalis $+C$ trachomatis. $\dagger \geqslant 5 \mathrm{PMNs} / \mathrm{HPF}$ and excludes cases with $T$ vaginalis, $N$ gonorrhoeae, $C$ trachomatis.

$\ddagger$ One or more of the following: $T$ vaginalis $+/-N$ gonorrhoeae $+/-C$ trachomatis $+/-\geqslant 5 \mathrm{PMNs} / \mathrm{HPF}$.

$N$ gonorrhoeae, $C$ trachomatis, or $T$ vaginalis were positive and to have non-specific urethritis (NSU) if they had at least five polymorphonuclear leucocytes per high power film on the urethral smear and were not infected with any of the above pathogens. Men were considered to have urethritis if they had a confirmed urethral infection or NSU.

\section{STATISTICAL ANALYSIS}

Data were compiled in Dbase IV (Ashton Tate) and data analysis was performed using STATA 5.0 software (College Station, TX, USA). Only men in whom all laboratory results were available were considered in the analysis. To test the associations of various factors and infection with $T$ vaginalis proportions were compared using $\chi^{2}$ analysis and odds ratios and their $95 \%$ confidence intervals were calculated for each risk factor and were adjusted for age in years using logistic regression. Multivariate analysis was used to examine variables associated with trichomoniasis on univariate analysis and to determine factors independently associated with $T$ vaginalis infection.

\section{ETHICAL ISSUES}

Ethical clearance was obtained from the Tanzanian National Institute for Medical Research, the London School of Hygiene and Tropical Medicine and the ethics committee of the Institute of Tropical Medicine in Antwerp. Informed consent was obtained from all participants who signed or placed a fingerprint on the consent form after the aims of the study had been explained to them.

\section{Results}

SOCIODEMOGRAPHIC CHARACTERISTICS

A total of 1298 men aged $15-54$ years living within the study area were eligible for inclusion and, of these, $1004(77 \%)$ were enrolled into the study. The main reason for nonparticipation in the study was travel out of the area. Two men refused the clinical examination and collection of urethral swabs. Complete laboratory results were available for 980 men $(98 \%)$. The mean age of this study population was 30.5 years (SD 11.2), 18\% were aged less than 20 years and $21 \%$ reported having received no education. The main occupation was farming $(79 \%)$ and $90 \%$ were from the Sukuma ethnic group. Of the 545 (56\%) mar- ried men, $46(8 \%)$ were in polygamous marriages. Sixty five individuals $(7 \%)$ denied any previous sexual activity.

PREVALENCE OF URETHRITIS AND

SCHISTOSOMIASIS

The prevalence of the different STDs and schistosomiasis in the study participants, stratified by age, is shown in table 1. Evidence of laboratory proved urethritis (NSU or infection with $T$ vaginalis, $C$ trachomatis, or $N$ gonorrhoeae) was found in $249(25 \%)$ men. $T$ vaginalis was the most prevalent specific STD pathogen identified, being isolated in 109 $(11 \%)$ on the basis of a positive wet preparation or culture. Twenty two men $(2 \%)$ had infection with either $C$ trachomatis or $N$ gonorrhoeae and $124(13 \%)$ had NSU. There were seven men with mixed infections; one gonococcal/ chlamydial infection, three gonococcal/T vaginalis infections, and three chlamydial/T vaginalis infections. Schistosoma haematobium infection was diagnosed in $364(37 \%)$ of the study population.

PREVALENCE OF STD SYMPTOMS AND SIGNS

Full information on symptoms and signs was collected on 978 of the 980 men who had complete laboratory results and further analysis is restricted to this subsample. Overall, 352 men $(36 \%)$ reported symptoms of dysuria, discharge, or scrotal tenderness or swelling to the interviewer or clinician and 18 men $(2 \%)$ had a discharge on examination. Dysuria was the commonest complaint, reported in 340/978 (35\%) individuals compared to the symptom of urethral discharge, the entry point for syndromic management of urethritis, which was only reported by 27 men (3\%). Of the 352 symptomatic men, 204 (58\%) had taken action to alleviate their symptoms before recruitment in the study. The commonest initial action was attendance at the health centre $(47 \%)$.

Table 2 shows the reported symptoms and clinical signs by infection in men with $T$ vaginalis alone, $N$ gonorrhoeae and/or $C$ trachomatis, and NSU compared with men with no laboratory evidence of urethral infection. Over half of the men with proved trichomoniasis (51\%) or $N$ gonorrhoeae and/or $C$ trachomatis infection $(62 \%)$ had no symptoms or signs of infection. In individuals without urethritis there was a high prevalence of symptoms and/or signs $(254 / 730 ; 35 \%)$ which were 
Table 2 Symptoms and signs in men by aetiology of urethritis and by schistosomiasis

\begin{tabular}{|c|c|c|c|c|c|}
\hline & \multicolumn{5}{|c|}{ No (\%) with symptom or sign* } \\
\hline & $\begin{array}{l}\text { Tvaginalis } \\
(n=103)\end{array}$ & $\begin{array}{l}N \text { gonorrhoeae/ } C \\
\text { trachomatis }+/-T \\
\text { vaginalis }(n=21)\end{array}$ & $\begin{array}{l}\text { Non-specific } \\
\text { urethritisf }(n=124)\end{array}$ & $\begin{array}{l}\text { No urethritis } \neq \\
(n=730)\end{array}$ & Total \\
\hline \multicolumn{6}{|l|}{ Symptoms } \\
\hline Dysuria & $43(42 \%)$ & $7(33 \%)$ & $48(39 \%)$ & $242(33 \%)$ & 340 \\
\hline Discharge & $5(5 \%)$ & $2(10 \%)$ & $2(2 \%)$ & $18(3 \%)$ & 27 \\
\hline Scrotal pain/swelling & $4(4 \%)$ & $0(0 \%)$ & $0(0 \%)$ & $18(3 \%)$ & 22 \\
\hline Any symptom $+\dagger$ & $46(45 \%)$ & $7(33 \%)$ & $48(39 \%)$ & $251(34 \%)$ & 352 \\
\hline \multicolumn{6}{|l|}{ Signs $₫$} \\
\hline Discharge & $9(9 \%)$ & $1(5 \%)$ & $2(2 \%)$ & $6(1 \%)$ & 18 \\
\hline Scrotal tenderness/swelling & $1(1 \%)$ & $0(0 \%)$ & $0(0 \%)$ & $0(0 \%)$ & 1 \\
\hline Symptoms and/or signs & $50(49 \%)$ & $8(38 \%)$ & $49(40 \%)$ & $254(35 \%)$ & 361 \\
\hline \multirow[t]{3}{*}{ No symptoms or signs } & 53 & 13 & 75 & 476 & 617 \\
\hline & \multicolumn{2}{|c|}{ Schistosomiasis positive } & \multicolumn{2}{|c|}{ Schistosomiasis negative } & \\
\hline & $\begin{array}{l}\text { Urethritis } \uparrow \\
(n=89)\end{array}$ & $\begin{array}{l}\text { No urethritis } \neq \\
(n=273)\end{array}$ & $\begin{array}{l}\text { Urethritis } \uparrow \\
(n=159)\end{array}$ & $\begin{array}{l}\text { No urethritis } \neq \\
(n=457)\end{array}$ & \\
\hline Symptoms and/or signs & $48(54 \%)$ & $135(50 \%)$ & $59(37 \%)$ & $119(26 \%)$ & 361 \\
\hline
\end{tabular}

significantly associated with the presence or absence of Schistosoma haematobium infection $\left(50 \%\right.$ \% 26\% respectively; $\left.\chi^{2}=41.3 ; \mathrm{p}<0.001\right)$. The most prevalent STD pathogen, $T$ vaginalis, was isolated in $14 \%$ of the 361 men with urethral symptoms or signs and in $9 \%$ of 617 men who had no symptoms or signs (adjusted for age; $\chi^{2}=8.57 ; \mathrm{p}=0.003$ ).

Of the 248 men with laboratory proved urethritis, 107 (43\%) had symptoms and/or signs of infection compared with 254 of 730 men (35\%) with no evidence of urethritis (OR 1.44; 95\% CI 1.08-1.94; $\mathrm{p}=0.014)$. However, when men were stratified by the presence or absence of schistosomiasis, symptoms and/or signs were no longer significantly associated with urethritis in those who had $S$ haematobium infection $\left(\chi^{2}=0.68 ; \mathrm{p}=0.41\right)$ although they were still significantly associated with urethritis in men without schistosomiasis $\left(\chi^{2}=7.29 ; p=0.007\right)$. Similar findings were observed for the association of symptoms and/or signs with trichomoniasis when stratified by schistosomiasis (data not shown).

For 103 men with $T$ vaginalis infection as the sole pathogen isolated, 46 (45\%) complained of symptoms suggestive of urethritis (dysuria, discharge, or scrotal swelling and tenderness). Dysuria was the commonest symptom, reported in $42 \%$. Only five men (5\%) with trichomoniasis complained of urethral discharge and four of scrotal pain or swelling. Urethral discharge was seen on examination in only $9 \%$ of men with trichomoniasis, $2 \%$ of men with NSU, and $5 \%$ of $N$ gonorrhoeae and/or $C$ trachomatis infections. There was no significant difference in the prevalence of signs and symptoms between men with $T$ vaginalis alone, men with gonorrhoea and/or chlamydial infection, and men with NSU $\left(\chi^{2}=\right.$ $0.83, \mathrm{p}=0.66$ ).

Twenty four of the 617 men without symptoms or signs of urethritis were wet preparation negative but had trichomoniasis identified by culture and were therefore not treated with metronidazole at the first visit. Nineteen of these men were seen at the 2 week follow up visit when they attended to collect their results and treatment. All denied developing symptoms of urethritis during the preceding 2 weeks although, at the follow up visit, four $(21 \%)$ who had no evidence of infection with any other pathogen had urethral discharge on examination.

DURATION OF INFECTION

It was not considered appropriate to ask men details about the specific date of last sexual intercourse. However, 11/108 (10\%) men with trichomoniasis denied having any sexual intercourse within the preceding 3 months and $9 / 108(8 \%)$ denied any intercourse within the past year. None of the eight men with gonococcal infection and $2 / 15(13 \%)$ of men with $C$ trachomatis denied sexual intercourse within the past 3 months.

\section{DIAGNOSIS OF TRICHOMONIASIS}

Two methods were used for the diagnosis of $T$ vaginalis. Wet mount preparation of urine sediment detected 71/108 (66\%) men diagnosed with trichomoniasis compared to culture which detected $68(63 \%)$ infections. Thirty seven positive cultures $(54 \%)$ were negative on wet preparation and $40(56 \%)$ wet preparations positive for $T$ vaginalis were culture negative. If the diagnosis had been based on only one of these methods then the prevalence would have been approximately $7 \%$. Furthermore, if the cultures had only been read at 3 days, rather than at both 3 days and 7 days, 15/68 (22\%) of the culture positive specimens would have been missed.

\section{ASSOCIATION WITH URETHRAL}

POLYMORPHONUCLEAR CELLS

There was only a weak association between the presence of polymorphs in the urethral smear and trichomoniasis. Twenty two of 103 men 
Table 3 LED test performance for detecting TV among 833 men with and without symptoms and signs depending on presence of schistosomiasis *

\begin{tabular}{|c|c|c|c|c|c|}
\hline & \multicolumn{2}{|l|}{ No (\%) LED+ } & \multirow[b]{2}{*}{ Sensitivity } & \multirow[b]{2}{*}{ Specificity } & \multirow{2}{*}{$\begin{array}{l}\text { Positive } \\
\text { predictive value }\end{array}$} \\
\hline & $T$ vaginalis + & T vaginalis - & & & \\
\hline \multicolumn{6}{|l|}{ Schistosomiasis } \\
\hline Symptoms and/or signs & $14 / 16(88 \%)$ & $87 / 135(64 \%)$ & $88 \%$ & $36 \%$ & $14 \%$ \\
\hline No symptoms/signs & $8 / 13(62 \%)$ & $76 / 138(55 \%)$ & $62 \%$ & $45 \%$ & $10 \%$ \\
\hline \multicolumn{6}{|l|}{ No schistosomiasis } \\
\hline Symptoms and/or signs & $26 / 34(76 \%)$ & $44 / 119(37 \%)$ & $76 \%$ & $63 \%$ & $37 \%$ \\
\hline No symptoms/signs & $24 / 40(60 \%)$ & $77 / 338(23 \%)$ & $60 \%$ & $77 \%$ & $24 \%$ \\
\hline \multicolumn{6}{|l|}{ All men } \\
\hline Symptoms and/or signs & $40 / 50(80 \%)$ & $131 / 254(52 \%)$ & $80 \%$ & $48 \%$ & $23 \%$ \\
\hline No symptoms/signs & $32 / 53(60 \%)$ & $153 / 476(32 \%)$ & $60 \%$ & $68 \%$ & $17 \%$ \\
\hline Total & $72 / 103(70 \%)$ & $284 / 730(39 \%)$ & $70 \%$ & $61 \%$ & $20 \%$ \\
\hline
\end{tabular}

$\star$ Excludes 147 men for whom either full data on the questionnaire was not available or who had $N$ gonorrhoeae or $C$ trachomatis or NSU.

with $T$ vaginalis infection as the sole pathogen isolated had $\geqslant 5$ PMN per high power field compared with 124 of 855 men who were not infected with $C$ trachomatis, $T$ vaginalis, or $N$ gonorrhoeae $\left(21 \%\right.$ and $15 \%$ respectively; $\chi^{2}$ 3.36; $\mathrm{p}=0.07)$. Similarly, there was a borderline association between urethral polymorphs and infection with $C$ trachomatis and/or $N$ gonorrhoeae $\left(31.2 \%\right.$ v $\left.15 \% ; \chi^{2} 3.49 ; \mathrm{p}=0.06\right)$.

Table 4 Factors associated with trichomoniasis among 978 rural men, Mwanza

\begin{tabular}{|c|c|c|c|c|c|}
\hline Characteristic & $\mathrm{No}^{\star}$ & $\begin{array}{l}\text { No with } T \\
\text { vaginalis }\end{array}$ & $\%$ & $\begin{array}{l}\text { Odds ratio } \\
(95 \% \mathrm{CI})\end{array}$ & p Value \\
\hline \multicolumn{6}{|l|}{ Education } \\
\hline None/adult only & 209 & 33 & 15.8 & 1.0 & \multirow[t]{4}{*}{$0.06+$} \\
\hline Standard 1-4 & 192 & 25 & 13.0 & $0.79(0.45-1.40)$ & \\
\hline Standard $\geqslant 5$ & 550 & 48 & 8.7 & $0.63(0.38-1.04)$ & \\
\hline Secondary & 27 & 2 & 7.4 & $0.47(0.10-2.08)$ & \\
\hline \multicolumn{6}{|l|}{ Employment } \\
\hline Various $\ddagger$ & 193 & 7 & 3.6 & 1.0 & \multirow[t]{3}{*}{$<0.001$} \\
\hline Farmer & 771 & 98 & 12.7 & $3.41(1.55-7.50)$ & \\
\hline Unskilled manual & 14 & 3 & 21.4 & $8.97(2.00-40.12)$ & \\
\hline \multicolumn{6}{|l|}{ Religion } \\
\hline Muslim & 125 & 3 & 2.4 & 1.0 & \multirow[t]{4}{*}{$<0.001$} \\
\hline Catholic & 381 & 43 & 11.3 & $4.96(1.51-16.32)$ & \\
\hline Protestant & 154 & 6 & 3.9 & $1.56(0.38-6.39)$ & \\
\hline Other & 318 & 56 & 17.6 & $7.48(2.28-24.53)$ & \\
\hline \multicolumn{6}{|l|}{ Travel in past year } \\
\hline No & 311 & 36 & 11.6 & 1.0 & \multirow{2}{*}{0.84} \\
\hline Yes & 667 & 72 & 10.8 & $0.96(0.62-1.48)$ & \\
\hline \multicolumn{6}{|l|}{ Marital status } \\
\hline Unmarried & 433 & 29 & 6.7 & 1.0 & \multirow[t]{3}{*}{$<0.001 \dagger$} \\
\hline Only wife & 499 & 67 & 13.4 & $2.08(1.14-3.82)$ & \\
\hline $2+$ wives & 46 & 12 & 26.1 & $4.35(1.81-10.44)$ & \\
\hline \multicolumn{6}{|c|}{ Lifetime sexual partners } \\
\hline $0-1$ & 114 & 6 & 5.3 & 1.0 & \multirow[t]{4}{*}{0.36} \\
\hline $2-5$ & 254 & 25 & 9.8 & $1.58(0.59-4.22)$ & \\
\hline $6-10$ & 215 & 30 & 14.0 & $2.09(0.76-5.73)$ & \\
\hline$>10$ & 395 & 47 & 11.9 & $1.45(0.53-4.01)$ & \\
\hline \multicolumn{6}{|c|}{ No of partners past 1 year } \\
\hline 0 & 131 & 9 & 6.9 & 1.0 & \multirow[t]{3}{*}{$0.28+$} \\
\hline 1 & 392 & 45 & 11.5 & $1.3(0.59-2.86)$ & \\
\hline$\geqslant 2$ & 455 & 54 & 11.9 & $1.50(0.69-3.27)$ & \\
\hline \multicolumn{6}{|c|}{ No. partners past 3 months } \\
\hline 0 & 215 & 11 & 5.1 & 1.0 & \multirow[t]{3}{*}{$0.001 \dagger$} \\
\hline 1 & 551 & 63 & 11.4 & $2.01(0.99-4.13)$ & \\
\hline$\geqslant 2$ & 212 & 34 & 16.0 & $3.25(1.51-6.98)$ & \\
\hline \multicolumn{6}{|l|}{ Circumcised $\$$} \\
\hline No & 773 & 98 & 12.7 & 1.0 & \multirow[t]{2}{*}{0.001} \\
\hline Yes & 202 & 10 & 5.0 & $0.37(0.19-0.72)$ & \\
\hline \multicolumn{6}{|c|}{$N$ gonorrhoeae $+/-C$ trachomatis infection } \\
\hline No & 957 & 103 & 10.8 & 1.0 & \multirow[t]{2}{*}{0.11} \\
\hline Yes & 21 & 5 & 23.8 & $2.53(0.89-7.18)$ & \\
\hline \multicolumn{6}{|c|}{ Genital ulceration on examination } \\
\hline Yes & 968 & 105 & 10.9 & 1.0 & \multirow[t]{2}{*}{0.085} \\
\hline No & 10 & 3 & 30 & $3.87(0.96-15.62)$ & \\
\hline
\end{tabular}

*Odds ratio (OR) controlled for age; excludes 2 men for whom full questionnaire information was not available.

qLikelihood ratio test for association.

†Likelihood ratio test for trend.

‡Includes skilled manual, office worker, teacher, business, fishing, truck drivers/turn boys and students.

\Excludes 3 men for whom data on circumcision status are missing.
PERFORMANCE OF THE LED TEST FOR

TRICHOMONIASIS

Of the 980 men with complete laboratory results 419 (43\%) had a positive LED test (defined as greater than "trace"). The performance of the LED test to detect $T$ vaginalis infection is shown in table 3, which compares LED results for men with trichomoniasis but no other pathogen or NSU and men who had no laboratory evidence of urethritis. Although a positive LED test was associated with $T$ vaginalis $\left(\chi^{2} 31.64 ; \mathrm{p}=<0.001\right)$ the performance of LED as a screening test for detecting trichomoniasis was poor with an overall sensitivity and positive predictive value of $70 \%$ and $20 \%$ respectively. A higher proportion of men with symptoms and/or signs were LED positive compared with asymptomatic men (56\% v 35\%; $\left.\chi^{2} 4.18, \mathrm{p}=0.041\right)$. In asymptomatic men the test missed $40 \%$ of infections and the positive predictive value was only $17 \%$. Because there was also a strong association between a positive LED test and infection with $S$ haematobium in this population $\left(\chi^{2} 20.54\right.$; $p$ $<0.001)$, the performance of the LED test for detecting $T$ vaginalis in men with and without urethral symptoms and signs is shown stratified by the presence or absence of schistosomiasis. In all groups the sensitivity and positive predictive value were better in symptomatic men, while the specificity was highest in asymptomatic men and men without schistosomiasis.

RISK FACTORS FOR TRICHOMONIASIS

Since there is little information on risk factors for $T$ vaginalis infection in men from subSaharan Africa we examined several sociodemographic, behavioural, and physical characteristics of men in our study population. The prevalence of infection increased with rising age (table 1), from $7 \%$ in men aged 15-19 years up to $17 \%$ in $35-44$ years $(p=0.002$ for trend). Data from two men were omitted from further analysis because their questionnaire data were incomplete.

Five sociodemographic and physical findings were significantly associated with $T$ vaginalis infection on univariate analysis after controlling for age (table 4). These included marital status, religion, job held, the number of sexual partners in the past 3 months, and being uncircumcised. Prevalence of infection was highest 
Table 5 Adjusted association of risk factors for trichomoniasis

\begin{tabular}{|c|c|c|c|}
\hline Characteristic & $\begin{array}{l}\text { Adjusted } \\
\text { odds ratio* }\end{array}$ & $95 \%$ CIS & $p$ Value \\
\hline \multicolumn{4}{|l|}{ Education } \\
\hline None/adult only & 1.0 & & 0.911 \\
\hline Standard 1-4 & 0.84 & $0.47-1.52$ & \\
\hline Standard $\geqslant 5$ & 0.92 & $0.52-1.61$ & \\
\hline Secondary & 1.36 & $0.27-6.94$ & \\
\hline \multicolumn{4}{|l|}{ Employment } \\
\hline Various $\ddagger$ & 1.0 & & 0.024 \\
\hline Farmer & 2.16 & $0.93-4.98$ & \\
\hline Unskilled manual & 8.95 & $1.82-43.92$ & \\
\hline \multicolumn{4}{|l|}{ Religion } \\
\hline Muslim & 1.0 & & $<0.001$ \\
\hline Catholic & 3.28 & $0.90-11.97$ & \\
\hline Protestant & 0.96 & $0.21-4.31$ & \\
\hline Other & 4.25 & $1.12-16.10$ & \\
\hline \multicolumn{4}{|l|}{ Marital status } \\
\hline Unmarried & 1.0 & & 0.023 \\
\hline Only wife & 1.78 & $1.02-3.11$ & \\
\hline $2+$ wives & 3.15 & $1.36-7.44$ & \\
\hline \multicolumn{4}{|c|}{ No of partners past 3 months } \\
\hline 0 & 1.0 & & 0.303 \\
\hline 1 & 1.47 & $0.67-3.21$ & \\
\hline$\geqslant 2$ & 1.90 & $0.82-4.37$ & \\
\hline \multicolumn{4}{|l|}{ Circumcised } \\
\hline No & 1.0 & & 0.409 \\
\hline Yes & 0.73 & $0.34-1.56$ & \\
\hline \multicolumn{4}{|l|}{ Genital ulceration } \\
\hline No & 1.0 & & \\
\hline Yes & 2.57 & $0.60-11.00$ & 0.228 \\
\hline
\end{tabular}

${ }^{\star} \mathrm{OR}$ adjusted for age in years, religion, marital status, employment, and circumcision.

SCI: $95 \%$ confidence interval.

¥Includes skilled manual, office worker, teacher, business, fishing, truck drivers/turn boys, and students.

in men who were married and who had two or more wives, men who had no established religion or who held traditional beliefs, men who were farmers or who worked as unskilled labourers, and those who had two or more sexual partners in the preceding 3 months. Circumcised men were significantly less likely to be infected (OR 0.37; $\mathrm{p}=0.001$ ). There was a lower prevalence of $T$ vaginalis infection with increasing level of education which reached borderline significance after adjustment for age ( $\mathrm{p}=0.055$ for trend). The prevalence of trichomoniasis was higher in those with a genital ulcer on examination, and this association was also of borderline statistical significance (OR 3.87; $\mathrm{p}=0.085$ ). Travel out of Misungwi in the past year, the number of sexual partners in the preceding 12 months and concurrent infection with $N$ gonorrhoeae or $C$ trachomatis were not significantly associated with trichomoniasis.

Variables associated with $T$ vaginalis on univariate analysis were examined using multiple logistic regression (table 5). Religion, being in a monogamous or polygamous marriage, and being an unskilled manual labourer were all independently associated with infection. The association of religion persisted after adjusting for circumcision but circumcision itself, education, genital ulceration on examination, and the number of partners in the preceding 3 months were no longer significantly associated with $T$ vaginalis infection in the multivariate analysis.

\section{Discussion}

Few population based studies to assess the prevalence of STDs have been conducted in Africa. The prevalence of gonorrhoea and of chlamydial infection found in this study are in line with the results of previous studies in Mwanza Region ${ }^{6}$ and Rakai District, Uganda. ${ }^{15}$ In the latter study $0.9 \%$ of men in the general population were found to have gonorrhoea and $2.1 \%$ to have chlamydial infection. We found that more than half of men with gonorrhoea or chlamydial infection were asymptomatic, similar to Rakai where $53 \%$ of men with gonorrhoea and $92 \%$ of men with chlamydial infection were asymptomatic.

Most studies on urethritis in men in Africa have focused on gonorrhoea and chlamydial infection. There has been very little information on $T$ vaginalis infection in African men, although $T$ vaginalis is believed to be one of the most prevalent STDs worldwide. ${ }^{16}$ This has generally been based on studies in women. Infection rates with trichomoniasis in subSaharan African women are among the highest in the world, with a WHO estimated prevalence of trichomonal infection of $14 \% .{ }^{16}$ Community based studies in Tanzania and Uganda have found a prevalence of $25 \%$ and $24 \%$, respectively, of trichomoniasis in rural women from the general population. ${ }^{15}{ }^{17}$

This study found $T$ vaginalis to be the most common STD pathogen identified in urethral samples from men in the general population. We demonstrated that trichomoniasis is highly prevalent in both symptomatic and asymptomatic men (14\% and $9 \%$ respectively). Two other studies that had included trichomoniasis in the assessment of male urethritis also found $T$ vaginalis to be an important pathogen. In Malawi it was identified in $16 \%$ and $9 \%$ of symptomatic male STD clinic attenders and asymptomatic men presenting to a skin clinic. ${ }^{18}$ Similarly $T$ vaginalis was isolated in $15 \%$ of symptomatic and $5 \%$ of asymptomatic transport workers in Kenya. ${ }^{11}$

It is likely in many studies that the prevalence of trichomonal infection in men has been underestimated. The study of Kenyan truck drivers demonstrated a prevalence of $6 \%$ using trichomonal culture alone. ${ }^{11}$ The higher prevalence of $T$ vaginalis in our study may be due in part to using two methods of diagnosis. Studies in pregnant women have found that a combination of wet mounts and culture are required to effectively detect $T$ vaginalis ${ }^{1920}$ although culture is generally taken to be the gold standard diagnostic method. ${ }^{21}$ The number of wet mount positive, culture negative samples in our study was higher than other studies where all or most wet mount positives are detected by culture. ${ }^{19}{ }^{22-24}$ This may reflect the fact that cultures were taken from urine sediment which is a less sensitive method than cultures from the urethra $^{2125}$ and also the field conditions under which cultures were taken and kept until they could be transported to the reference laboratory in Mwanza. However, the use of both methods approximately doubled the number of infections identified in this population than if only one method had been used. Allowing at least 1 week for incubation of trichomonal cultures, which has been noted by other investigators, ${ }^{25}$ was also important in this study given the fact that $22 \%$ of positive cultures were only diagnosed after 7 days' incubation. 
Given the poor concordance of the two tests it is likely that the observed prevalence of trichomoniasis may also be an underestimate. From the proportion of total positive cases missed by each test it is possible to calculate an estimate of the number of $T$ vaginalis cases missed by both tests $\left(\right.$ as $\left(1-p_{1}\right)\left(1-p_{2}\right)$ where $\mathrm{p}_{\mathrm{x}}$ is the probability of a true case being detected by each test, assuming the two tests are independent). The proportion of trichomoniasis cases potentially missed by both tests is $12.6 \%$, an additional 16 cases which would give a maximum theoretical prevalence of approximately $13 \%$.

Only three independent factors were found to be associated with trichomoniasis. Religion, marital status, especially being in a polygamous marriage, and working as an unskilled manual labourer were all associated with an increased risk of trichomoniasis. The $23 \%$ of men who were not enrolled in the study because they had travelled out of the area may have been at higher risk of trichomonal or other urethral infections. However, a history of travel within the past year was not associated with trichomoniasis in men included in the study. In this population circumcision was associated with a lower risk of trichomoniasis on univariate analysis but it was not found to be an independent risk factor after adjustment for religion and employment. The number of sexual partners in the past 3 months was associated with a higher risk of trichomoniasis in univariate analysis, but the association did not persist in multivariate analysis. Although nonchlamydial non-gonococcal urethritis was associated with trichomoniasis in male STD clinic attenders in the United States, ${ }^{25}$ no independent association was found between $T$ vaginalis and other STDs, similar to the findings in our study. This, together with the fact that there was no association between risk of trichomoniasis and number of sex partners in the past 3 months, suggests that $T$ vaginalis infection may have different transmission dynamics from the other STDs. More research is needed on the epidemiology and risk factors of trichomoniasis in Africa.

Rapid, spontaneous resolution of trichomoniasis has been reported, ${ }^{26}{ }^{27}$ but from observational studies and human inoculation experiments trichomonads have been known to persist in the male urethra in untreated men for up to 101 days. $^{26}{ }^{28}$ In this study $8 \%$ of men with trichomoniasis who denied sexual intercourse for up to 12 months had evidence of viable trichomonads on culture or wet preparation. Although these data on sexual behaviour should be interpreted with some caution, they do suggest that trichomoniasis is a persistent infection in some individuals. As Kreiger has pointed out, the consequences of chronic infection with $T$ vaginalis in men are unclear although the asymptomatic carrier state is known to be relatively common. ${ }^{21}$ It is possible that trichomoniasis may be associated with a relative lack of inflammation in the urethra since it was not significantly associated with the presence of $\geqslant 5$ polymorphonuclear cells per high power field in men who had no evidence of infection with another STD pathogen. Among men with $T$ vaginalis as the sole pathogen identified, only $21 \%$ had $\geqslant 5$ polymorphonuclear cells per high power field.

The high prevalence of trichomoniasis in men in east Africa found in this and other studies has several important implications. Firstly, trichomoniasis has been associated with an increased risk of acquiring HIV infection in commercial sex workers in Congo (formerly Zaire) $)^{3}$ and with preterm delivery and low birth weight in pregnant women. ${ }^{29}$ Hobbs et al in Malawi found that men with symptomatic trichomoniasis had higher median concentrations of HIV RNA in semen compared with controls without any STD pathogen or men with asymptomatic trichomonas infection. This suggests that trichomoniasis in men may be associated with a higher level of infectiousness if it is also associated with inflammation of the urethra. Whether trichomoniasis increases the susceptibility to HIV infection of men remains so far unclear.

Secondly, men complaining of urethral symptoms who have access to a health facility would currently be managed syndromically. ${ }^{30}$ Current first line recommendations do not cover treatment for $T$ vaginalis infection. Given the findings of this and other studies ${ }^{11} 18{ }^{31}$ there is a need to measure the prevalence and investigate the role of trichomoniasis in symptomatic men presenting to health facilities for the syndromic management of urethritis and to consider including metronidazole as one of the first line treatments for urethritis. Furthermore, over $50 \%$ of the men harbouring $T$ vaginalis had no symptoms or signs of infection and would therefore also remain as potential reservoirs of infection in the community. Data from the follow up visit from asymptomatic men with trichomoniasis who were not treated at the first screening visit suggest that, in the men who did not go on to develop symptoms in the intervening period, the infections in those individuals were truly asymptomatic.

Diagnosing trichomoniasis and other causes of urethritis using laboratory methods such as Gram stains and culture of urethral swabs is unrealistic for many developing countries. The presence of urethral symptoms or signs as a marker for urethritis is problematic in this population where $57 \%$ of men with laboratory proved urethritis had clinically silent infections and where $34 \%$ of men with no evidence of urethritis and $26 \%$ of men without schistosomiasis or urethritis complained of urethral symptoms. Urethral symptoms are particularly likely to be poor predictors of urethritis in populations where urinary schistosomiasis is endemic, especially in countries which have a similar high prevalence of $S$ haematobium. ${ }^{32}$ Many of the men with urethral symptoms but no confirmed urethritis in this study were in fact suffering from schistosomiasis. If trichomoniasis has a role in morbidity and, in addition to other non-ulcerative STDs, is a risk factor for enhancing HIV transmission, ${ }^{3}$ then screening tests that would help to identify asymptomatic infections when men do present to the health services are urgently needed. One 
of the tests that has been suggested is the LED test because it is cheap, easy to use and can give an immediate result. The sensitivity and specificity of LED for detecting trichomoniasis proved disappointing in this population. There remains an urgent requirement for the development of simple, affordable and reliable screening tests to diagnose asymptomatic STDs both in women and men. ${ }^{9} 3334$

Conflict of interest: none declared.

Funding: This study was funded by the Commission of the European Community (DG XII), and investigators were supported by the Wellcome Trust, London (DWJ), the Overseas Development Administration of the United Kingdom (PM, BW), the Commission of the European Community DG VIII (JT), and the Centre of International Migration and Development of the Federal Government of Germany (HG)

The authors thank the principal secretary, Ministry of Health, and the director general of the National Institute of Medica Research for permission to carry out and publish the results of this study. The support of the Misungwi government officials is gratefully acknowledged and we are grateful to the hospitality of the individuals who participated in the survey. We would also like to thank Mary Shushu, Frexon Lisekie, and Tamara Hurst for their valuable assistance.

Contributors: DWJ developed the questionnaires and field protocol, was responsible for field study organisation and supervision, data analysis and manuscript preparation; KM supervised the field study teams and recruitment; PM assisted in the study design, developed the questionnaires and protocol, was responsible for field study organisation and supervision and manuscript preparation; $\mathrm{LN}$ supervised the field study teams and recruitment; JT assisted in the study design, developed the questionnaires and was in charge of statistical analysis; FM was responsible for field study organisation and supervision; BW assisted in the study design and supervised the laboratory testing; $\mathrm{BCF}$ was responsible for follow up of the study participants HG developed the study design and manuscript preparation ML developed the study design and protocol; RH developed the study design and manuscript preparation; DM developed the study design and manuscript preparation; $\mathrm{AB}$ was the primary
investigator, developed the study design, protocol, and assisted in the data analysis and manuscript preparation.

1 Plummer FA, Simonsen JN, Cameron DW, et al. Cofactors Plummer FA, Simonsen JN, Cameron DW, et al. Cofactors in male-female sexual transmission of human imm

2 Fleming DT, Wasserheit JN. From epidemiological synergy to public health policy and practice: the contribution of other sexually transmitted diseases to sexual transmission of HIV infection. Sex Transm Inf 1999;75:3-17.

3 Laga M, Manoka A, Kivuvu M, et al. Non-ulcerative sexually transmitted diseases as risk factors for HIV-1 transmission in women: results from a cohort study. AIDS 1993;7:92-102

4 Cohen MS. Sexually transmitted diseases enhance HIV transmission: no longer a hypothesis. Lancet 1998;351 (Suppl III): 5-7.

5 Grosskurth H, Mosha F, Todd J, et al. Impact of improved treatment of sexually transmitted disease on HIV infection in rural Tanzania:randomised control trial. Lancet 1995; 346:530-6.

6 Grosskurth H, Mayaud P, Todd J et al. Asymptomatic gonorrhoea and chlamydial infection in rural Tanzanian men. orrhoea and chlamydial

7 Mabey DCW. The diagnosis and treatment of urethritis in developing countries. Genitourin Med 1994;70:1-2.

8 Mayaud P, Changalucha J, Newell J, et al. The value of urine specimens in screening for male urethritis and it aetiologies in Tanzania. Genitourin Med 1992;68:361-5.

9 Mayaud P, ka-Gina G, Cornelissen J, et al. Validation of a WHO algorithm with risk assessment for the clinical management of vaginal discharge in Mwanza, Tanzania. Sex Transm Inf 1998;74 (Suppl 1): S77-84.

10 Mayaud P, Uledi E, Cornelissen J, et al. Risk scores to detect cervical infections in urban antenatal attenders in Mwanza, Tanzania. Sex Transm Inf 1998;74 (Suppl 1):S139-46.
11 Jackson DJ, Rakwar JP, Chohan B, et al. Urethral infection in a workplace population of East African men: evaluation in a workplace population of East African men: evaluation of strategies for

12 Vuylsteke B, Laga M, Alary M, et al. Clinical algorithms for the screening of women for gonococcal and chlamydial infection: evaluation of pregnant women and prostitutes in Zaire. Clin Inf Dis 1993;17:82-8.

13 Costello Daly C, Maggwa N, Mati JK, et al. Risk factors for gonorrhoea, syphilis and trichomonas infections among women attending family planning clinics in Nairobi. Genitourin Med 1994;66:62-5.

14 Tyndall M, Nasio J, Maitha G, et al. Leucocyte esterase urine strips for the screening of men with urethritis - use in developing countries. Genitourin Med 1992;68:361-5.

15 Wawer MJ, Sewankambo NK, Serwadda D, et al. Control of sexually transmitted diseases for AIDS prevention in Uganda: a randomised community trial. Lancet 1999;353: 525-35.

16 Gerbase AC, Rowley JT, Heymann DHL, Berkeley SFB, Piot P. Global prevalence and incidence estimates of selected curable STDs. Sex Transm Inf 1998;74 (Suppl 1):S12-16.

17 Klouman E, Masenga EJ, Klepp K-I, et al. HIV and reproductive tract infections in a total village population in rural Kilimanjaro, Tanzania. F Acquir Immune Defic Syndr 1997; 14:163-8.

18 Hobbs MM. Kazembe P, Reed AW, et al. Trichomonas vaginalis as a cause of urethritis in Malawian men. Sex Transm Dis 1999;26:381-7.

19 Pastorek JG, Cotch MF, Martin DH, et al. Clinical and microbiological correlates of vaginal trichomoniasis during pregnancy. Clin Infect Dis 1996;23:1075-80.

20 Draper D, Parker R, Patterson E, et al. Detection of Trichomonas vaginalis in pregnant women with the InPouch TV culture system. F Clin Microbiol 1993;31: 1016-18

21 Kreiger JN. Trichomoniasis in men: old issues and new data. Sex Transm Dis 1995;22:83-6.

22 Borchardt KA, Hernandez V, Miller S, et al. A clinical evaluation of trichomoniasis in San Jose, Costa Rica, using the InPouch TV test. Genitourin Med 1992;68:328-30.

23 Borchardt KA, Al-Haraci S, Maida N, et al. Prevalence of Trichomonas vaginalis in a male sexually transmitted disease clinic population by interview, wet mount microscopy and the InPouch ${ }^{\mathrm{TM}} \mathrm{TV}$ test. Genitourin Med 1995;71: $405-6$.

24 Fouts AC, Kraus SJ. Trichomonas vaginalis: reevaluation of its clinical presentation and laboratory diagnosis. F Infect Dis 1980;141:137-43.

25 Kreiger J, Verdon M, Siegel N, et al. Risk assessment and laboratory diagnosis of trichomoniasis in men. F Infect Dis 1992;166:1362-6.

26 Whittington MJ. Epidemiology of infections with Trichomonas vaginalis in the light of improved diagnostic methods. Brit f Vener Dis 1957;33:80-8.

27 Weston TE, Nicol CS. Natural history of trichomonal infection in males. Br f Vener Dis 1963;39:251-7.

28 Lancely F, McEntegart MG. Trichomonas vaginalis in the male: the experimental inoculation of a few volunteers. Lancet 1953;1:668-71.

29 Cotch MF, Pastorek JG, Nugent RP, et al. Trichomonas vaginalis associated with low birth weight and preterm delivery. The Vaginal Infections and Prematurity Study Group. Sex Transm Dis 1997;24:353-60

30 World Health Organization. Management of sexually transmitted diseases. WHO/GPA/TEM 94.1. Geneva: WHO, 1994.

31 Dallabetta G, Behets F, Lule G, et al. Specificity of dysuria and discharge complaints and presence of urethritis in male 1998;74 (Suppl 1):S34-7.

32 Doumenge JP, Mott KE, Cheung C, et al. Atlas of the global distribution of schistosomiasis. World Health Organization, Presses Universitaires de Bordeaux, 1987.

33 Chernesky M. How can industry, academia, public health authorities and the Sexually Transmitted Diagnostics Initiative (SDI) work together to help control sexually transmitted diseases in developing countries? Genitourin Med 1997;73:1-2.

34 Alary M, Baganizi E, Guèdèmè $\mathrm{A}$, et al. Evaluation of clinical algorithms for the diagnosis of gonococcal and chlamydial infections among men with urethral discharge and women with vaginal discharge in Benin. Sex Transm Inf 1998;74 (Suppl 1):S44-9. 